\title{
Assessment of the effects of acute and repeated exposure to blast overpressure in rodents: toward a greater understanding of blast and the potential ramifications for injury in humans exposed to blast
}

\author{
Stephen Thomas Ahlers ${ }^{1}$ *, Elaina Vasserman-Stokes ${ }^{1}$, Michael Christopher Shaughness ${ }^{1}$, \\ Aaron Andrew Hall ${ }^{1}$, Debra Ann Shear ${ }^{2}$, Mikulas Chavko ${ }^{1}$, Richard Michael McCarron ${ }^{1}$ and \\ James Radford Stone ${ }^{3,4}$ \\ ${ }^{1}$ Department of Neurotrauma, Operational and Undersea Medicine Directorate, Naval Medical Research Center, Silver Spring, MD, USA \\ ${ }^{2}$ Department of Applied Neurobiology, Walter Reed Army Institute of Research, Silver Spring, MD, USA \\ ${ }^{3}$ Department of Radiology, University of Virginia, Charlottesville, VA, USA \\ ${ }^{4}$ Department of Neurosurgery, University of Virginia, Charlottesville, VA, USA
}

\section{Edited by:}

Ibolja Cernak, Johns Hopkins

University Applied Physics Lab, USA

Reviewed by:

Cameron Bass, Duke University, USA

Barclay Morrison, Columbia

University, USA

${ }^{*}$ Correspondence:

Stephen Thomas Ahlers, Operational and Undersea Medicine Directorate, Naval Medical Research Center, 503 Robert Grant Avenue, Silver Spring, MD 20910-74500, USA.

e-mail: stephen.ahlers@med.navy.mil
Mild traumatic brain injury (mTBI) resulting from exposure to improvised explosive devices (IEDs) has fueled a requirement to develop animals models that mirror this condition using exposure to blast overpressure (BOP). En route to developing a model of repeated exposure to BOP we sought to initially characterize the effects of acute BOP exposure in rodents, focusing specifically on the levels of BOP exposure that produced clinical mTBI symptoms. We first measured BOP effects on gross motor function on a balance beam. Separate groups of unanesthetized rats were exposed (in different orientations) to 36.6, 74.5, and $116.7 \mathrm{kPa}$ BOP exposure inside a pneumatically driven shock tube. Results demonstrated that rats exposed to $116.7 \mathrm{kPa}$ demonstrated transient alterations or loss of consciousness indicated by a transient loss of righting and by increased latencies on the balance beam. The $116.7 \mathrm{kPa}$ exposure was the threshold for overt pathology for acute BOP exposure with approximately $30 \%$ of rats presenting with evidence of subdural hemorrhage and cortical contusions. All animals exposed to $116.7 \mathrm{kPa}$ BOP manifested evidence of significant pulmonary hemorrhage. Anterograde memory deficits were observed in rats exposed to $74.5 \mathrm{kPa}$ facing the BOP wave and rats exposed to $116.7 \mathrm{kPa}$ in the lateral (side) orientation. We next assessed repeated exposure to either lateral or frontal $36.6 \mathrm{kPa}$ BOP in anesthetized rats, once per day for 12 days. Results showed that repeated exposure in the frontal, but not side, orientation to the BOP wave produced a transitory learning deficit on a Morris water maze task as shown by significantly longer latencies to reach the submerged platform in the second and third blocks of a four block session. Implications of these data are discussed in relation to the manifestation of $\mathrm{mTBI}$ in military personnel exposed to IEDs. Finally, we suggest that there are multiple types of long-term brain injury from blast exposure.

Keywords: blast injury, improvised explosive devices, traumatic brain injury, memory, pathology, orientation, loss or alteration of consciousness, concussion

\section{INTRODUCTION}

Significant attention has been focused on mild traumatic brain injury (mTBI) resulting from exposure to improvised explosive devices (IEDs; Okie, 2005; Warden and French, 2005; Warden, 2006; Hicks et al., 2010; Moore and Jaffee, 2010; Peskind et al., 2010; Snell and Halter, 2010). Studies of mTBI resulting from blast exposure occur within a broader context to understand the relationship between brain injury and blast exposures across the TBI spectrum (mild, moderate, and severe), with the aim to characterize underlying pathophysiological mechanisms and consequent neurological impairments. By far, the overwhelming representation of TBIs resulting from Operations Iraqi Freedom and Enduring
Freedom are mild and are presumed to result from one or more exposures to IEDs. IEDs also produce moderate and severe TBI that is often a component of polytrauma involving amputations, organ damage, and overwhelming systemic injury (Zouris et al., 2006; Bell et al., 2009). The conditions of blast exposure that produce mTBI in humans are not well understood. This is in part due to the complexity of the combat environment, with many IED exposures involving some combination of blast overpressure (BOP) exposure, blunt impact, and/or acceleration/deceleration inertial loading. Connecting a clinical diagnosis of mTBI to single or multiple traumatic exposures is further complicated by the subthe nature of the injury, concerns over adequate diagnostic criteria 
for mTBI, significant controversy related to the co-morbidity of post-traumatic stress disorder (PTSD) in the setting of mTBI, and presentation of post-concussive symptoms (PCS) long after the traumatic event. These issues underscore the need to more fully understand the relationship between exposure to blast events/blast components, and the neurological alterations culminating in a clinical manifestation of mTBI.

While clinical definitions of mTBI vary, they share a common outcome of an alteration in consciousness or cognition in less than $24 \mathrm{~h}$ following a traumatic event where there is no evidence of overt physical trauma using conventional neuroimaging or encephalographic techniques. The American Congress of Rehabilitation Medicine defines mTBI as a "traumatically induced physiological disruption of brain function manifested by either a loss of consciousness (LOC), loss of memory for events immediately before or after the accident, an alteration in mental state at the time of the accident (an alteration of consciousness or AOC), or a focal neurological deficit which may or may not be transient" [Developed by the Mild Traumatic Brain Injury Committee of the Head Injury Interdisciplinary Special Interest Group of the American Congress of Rehabilitation Medicine, 1993].

Several studies have assessed components of mTBI in military populations exposed to IEDs relying heavily upon retrospective self-assessment of loss or alteration of consciousness around of the time of the blast exposure, often many weeks or months after the event(s) (French and Parkinson, 2008; Galarneau et al., 2008; Gaylord et al., 2008; Hoge et al., 2008; Wilk et al., 2010). Many studies report long-term cognitive impairments that are often accompanied by headache, tinnitus, emotive changes, and broader range of PCS. These symptoms contribute to an even greater overlap, controversy, and to some extent confusion with PTSD (Hoge et al., 2008; Lew et al., 2009; Mora et al., 2009; Elder et al., 2010; Kennedy et al., 2010a,b; Lippa et al., 2010). While clarity of diagnosis and the underlying physiological mechanisms will undoubtedly take time to sort out, research to assess the short- and long-term consequences of exposure to IEDs is essential to elucidate the effects of acute and repeated exposure to blast events or components of blast such as BOP. To accomplish this, multiple animal models were developed to characterize underlying pathophysiological mechanisms and consequent neurological impairments from varying intensities of blast.

There are, at present, a significant number of studies that have demonstrated that exposure to native blast or BOP generated from a shock tube induces changes in behavior pathology, or both using animal models (Cernak et al., 2001, 2010; Moochhala et al., 2004; Long et al., 2009; Saljo et al., 2009, 2010b; Risling et al., 2010; Svetlov et al., 2010; Lu et al., 2011). The majority of studies with animal subjects have focused on the effects of a single blast exposure. Only recently has there been an emphasis on assessing the effects of repeated exposure to BOP (Saljo et al., 2010a). Though still in early the stages, evidence suggests that exposure to low BOP intensities can result in altered physiological, behavioral, inflammatory, and genomic status (Risling et al., 2010; Pun et al., 2011).

At present, individuals in both training and operational environments are routinely exposed to low-level blast in a fashion that would not necessarily prompt immediate medical attention. Exposures generally involve the blast wave alone without shrapnel or other secondary/tertiary forces. As an example, military breachers, use explosives in the operational or training environment to gain rapid entry into or across a hardened structure. Unpublished anecdotal reports from breacher populations suggest that extensive exposure to low-level blast events can result in enduring cognitive deficits. The increased awareness of reports of impairment from breacher populations coupled with the significant number of reported mTBI cases from casualties has prompted several studies of military breachers to assess the effects of exposure to a primary blast wave forces un-confounded by secondary and tertiary that are prevalent in battlefield environments. The purpose of this study was to systematically assess the neurological correlates of low-level primary blast exposure in a rodent model. This model has been designed to be conceptually similar to the breacher and operational populations similarly exposed to multiple IEDs or breaching blast events.

\section{MATERIALS AND METHODS ANIMALS AND BLAST EXPOSURE}

All studies with animal subjects were reviewed and approved by the Institutional Animal Care and Use Committee (IACUC). Adult male Long Evans Hooded rats (Charles River Laboratories International, Inc., Wilmington, MA, USA; 250-300 g) were used as subjects. Experimental overpressure exposure was performed using the Walter Reed Army Institute of Research (WRAIR) shock tube simulating effects of air blast exposure under experimental conditions. This shock tube has a $12^{\prime \prime}$ circular diameter, $19.5 \mathrm{ft}$. long steel tube divided into a $2.5 \mathrm{ft}$. air compression chamber separated from a $17 \mathrm{ft}$. expansion chamber by polyethylene Mylar ${ }^{\mathrm{TM}}$ sheets (Du Pont Co., Wilmington, DE, USA). The thickness of the Mylar sheets varied depending upon the peak pressure desired (Chavko et al., 2007; Elder et al., 2010). There were three BOP intensities for the current set of experiments corresponding to $36.6,74.5$, and $116.7 \mathrm{kPa}$.

The characteristics of the overpressure wave produced by the shock tube used in this study have been described extensively (Long et al., 2009; Chavko et al., 2011). The overpressure wave produced by the WRAIR shock tube exhibits waveform resembling a Friedlander wave form but with characteristics unique to its construction that do not reflect a strict Friedlander function. Shock tube characteristics differ in important ways that may affect the physiological and functional outcome resulting from exposure. Table 1 provides the defining characteristics of the WRAIR shock tube using the Mylar membranes that approximate the overpressure conditions referred to in this study. The reference "static" pressure inside the shock tube was measured using a piezoelectric sensor (PCB Piezotronics, Buffalo, NY, USA) placed between the rat head and the shock tube walls, approximately $3 \mathrm{~cm}$ from the head and $5 \mathrm{~cm}$ from the wall in a manner described by Chavko et al. (2011). The signal was recorded by the NI data acquisition system (National Instrument, Austin, TX, USA) at $500 \mathrm{kHz}$ sampling rate. Other important variables in determining the outcome are also provided in the table. These are measures of overpressure duration and the integral function reflecting the area under the overpressure wave. In general, duration and integral parameters increase as the peak pressure increases. 
Table 1 | Blast overpressure parameters: each data point represents the mean and SEM of five independent exposures.

\begin{tabular}{lll}
\hline $\begin{array}{l}\text { Maximum } \\
\text { overpressure (kPa) }\end{array}$ & $\begin{array}{l}\text { Overpressure } \\
\text { duration }(\mathbf{m s})\end{array}$ & $\begin{array}{l}\text { Overpressure } \\
\text { integral (kPa*ms) }\end{array}$ \\
\hline $36.6(1.6)$ & $4.1(0.3)$ & $75.2(4.5)$ \\
$74.5(4.5)$ & $4.8(0.2)$ & $175.8(11.1)$ \\
$116.7(7.0)$ & $6.8(0.2)$ & $335.5(8.5)$ \\
\hline
\end{tabular}

Pressure measurements were measured inside the shock tube using piezoelectric pressure sensors.

\section{ACUTE BOP EXPOSURE}

\section{Blast overpressure exposure}

Rats were exposed to either $0,36.6,74.5$, and $116.7 \mathrm{kPa}$ BOP. Sham $(0 \mathrm{kPa})$ rodents were prepared in same fashion as experimentally injured animals, without undergoing BOP exposure. For each blast level and for all experiments, separate groups of rats were exposed to BOP facing or sideways relative to the blast wave. In the initial experiments using the balance beam task we included a condition facing away from the blast wave but dropped this condition for subsequent studies based upon the lack of efficacy. Immediately prior to the BOP or sham exposure each rat was placed into a plastic cone-shaped rodent restraint cone (Harvard Apparatus, Holliston, MA, USA). After the rat was placed into the restraint cone the wide end of the cone was closed and secured using duct tape. The rat was then placed inside a cylindrical metal cage that extended $18^{\prime \prime}$ inside the shock tube. The restrained rat was held down in the metal cage by three bungee cords that restricted, but did not completely eliminate, movement. Within 20-30 s after the rat was placed into the shock tube the compressor was turned on until the Mylar membrane ruptured. Immediately after the BOP exposure rats were removed from the chamber and restraint device and placed into their home cage and observed for signs of impairment. The entire restraint/BOP exposure procedure was accomplished in approximately $1 \mathrm{~min}$.

The overarching focus of the studies was to mirror conditions experienced by operational forces. For this reason, we exposed rats to BOP without anesthesia for the acute studies. The lack of anesthetic use in these studies presented several experimental design considerations and an overall conservative and cautious stepwise approach. An important first question is the determination of the threshold BOP exposure that is cognitively debilitating versus a level that produces overt (and enduring) impairment of function? It is recognized that exposure to blast of the type that we intended to study may produce an acute concussive event and temporary debilitation. Often however, as with concussion in human populations, e.g., with sports injury, neurological disruption is transitory, and the individual recovers fully in a matter of minutes or hours. Our approach to the question of what level of blast would affect memory processes underscored the need for preliminary studies to determine a dose-response function to determine what level of blast exposure in unanesthetized animals results in outward signs of either permanent or transitory debilitation. In concert with the IACUC, the initial approach of acute BOP exposure entailed starting with the lowest exposure intensity $(36.6 \mathrm{kPa})$ and progressing to higher intensities of $74.5 \mathrm{kPa}$ and then $116.7 \mathrm{kPa}$ until a threshold of debilitation or untoward effects (e.g., hemorrhage) was observed. Once reaching this level we did not expose rats to higher intensities and subsequent studies focused on the effects of lower, non-pathologic exposures as the primary intent of the investigation was to elucidate the effects of low-level exposure to BOP similar to that is seen in operational breachers and military personnel exposed to repeated blast events. Our experiments demonstrated that the critical threshold for overt pathology was $116.7 \mathrm{kPa}$ where we began to see evidence of brain and lung pathology in rats.

\section{Balance beam task}

Three days before exposure to BOP rats were trained daily to walk the entire length of a $2-\mathrm{cm}$ (approximately $1^{\prime \prime}$ ) wide $\times 200 \mathrm{~cm}$ long wooden beam which was suspended $91.4 \mathrm{~cm}$ above the floor. On each trial the rat was released onto the end of the beam and is required to run down the entire beam and into the dark box. After adequate training, the average time for a 250- to 300-g adult rat to traverse the beam was 3-5s; each session was the average of two trials. Rats failing to traverse the beam in $15 \mathrm{~s}$ either from immobility or falling off the beam were given a $15 \mathrm{~s}$ score. Once stable performance was achieved for two consecutive sessions rats were randomly assigned to groups ( $n=6$ in each) and exposed to 0 , $36.6,74.5$, or $116.7 \mathrm{kPa}$ in the shock tube described above. After exposure to the BOP rats were assessed for performance on the balance beam $30 \mathrm{~min}, 2 \mathrm{~h}, 6 \mathrm{~h}, 24 \mathrm{~h}, 72 \mathrm{~h}$, and 1 week after exposure utilizing a within subject design. In addition, there were initially three orientation conditions: (1) facing the blast wave, (2) sideways ( side) orientation to the blast wave, and (3) facing away from the blast wave.

\section{Passive avoidance task}

The passive avoidance (PA) apparatus was a Plexiglas ${ }^{\circledR}$ shuttle box divided equally into two compartments. One compartment, including the lid, is painted black. The other compartment is painted white and has a clear Plexiglas ${ }^{\circledR}$ lid. A guillotine doorway connects the two compartments. The floor consists of stainless steel grids. A matched impedance shock source is connected to the grids on the dark side to which a very brief scrambled footshock $(0.4 \mathrm{~mA}$ for $1 \mathrm{~s})$ was delivered. Previous analysis had determined that $0.4 \mathrm{~mA}$ footshock was the minimum intensity to achieve onetrial learning. PA training consisted of placing the animal in the white side of the training apparatus facing toward the door. After $10 \mathrm{~s}$, the door was lifted and the rat had $60 \mathrm{~s}$ to cross the black side with all four paws. Rats failing to enter the dark compartment in $60 \mathrm{~s}$ were removed from the study. Immediately after crossing into the dark compartment the door was closed and a 1-s inescapable footshock was delivered. After delivery of the footshock, the animal was removed from the apparatus and returned to its home cage. Retention testing was identical to the training procedure except that no footshock was delivered. Rats are placed in the white side of the training apparatus and, after $10 \mathrm{~s}$, the door is opened and the latency to cross over to the black side as well as the total time spent on the white (safe) side (TTW) is recorded in a 10-min test session. Rats not crossing into the dark compartment in the 10-min test are given a latency and TTW score of $600 \mathrm{~s}$. 


\section{Retrograde amnesia studies}

To determine the retrograde influences of exposure to BOP, separate groups $(n=10)$ of rats were trained on the PA task then exposed to $0,36.6,74.5$, or $116.7 \mathrm{kPa} \mathrm{BOP}$ (random assignment) in either the sideways or facing orientations immediately (within $2 \mathrm{~min}$ ) after training. All animals were tested for retention $24 \mathrm{~h}$ after exposure to the BOP.

\section{Anterograde amnesia studies}

The design is similar to the retrograde amnesia studies except that training occurred after BOP exposure. Separate groups $(n=10)$ were trained on the PA task within 5 min after exposure to $0,36.6$, 74.5 , or $116.7 \mathrm{kPa} \mathrm{BOP}$ (random assignment) in either the sideways or facing orientations and assessed for retention $24 \mathrm{~h}$ after training. Because rats in the $116.7 \mathrm{kPa}$ demonstrated transient loco-motor impairment that affected their ability to walk through the shuttle box door, these animals were trained $20 \mathrm{~min}$ after BOP exposure.

\section{REPEATED BOP EXPOSURE}

For the repeated exposure to low-level $(36.6 \mathrm{kPa}) \mathrm{BOP}$ rats were anesthetized during the BOP exposures in order to reduce the inherent stress from repeated restraint and because the prior acute studies had determined that this level of exposure produced no observable untoward effects. Individual rats were anesthetized using an isoflurane gas anesthesia system consisting of a vaporizer, gas lines, and valves and an activated charcoal scavenging system adapted for use with rodents. Rats were placed into a polycarbonate induction chamber, which was closed and immediately flushed with $5 \%$ isoflurane mixture in air for $2 \mathrm{~min}$. In order to maintain consistency between studies we again placed rats into the plastic cone restraint device during the repeated BOP exposures. In addition, we modified the blast exposure parameters slightly to restrict movement during the blast exposure. This was accomplished using flattened rubber tourniquet tubing that was $1.5 \mathrm{~cm}$ in diameter. Three tourniquets were spaced evenly to secure the head region, the upper torso and lower torso regions while the animal was in the plastic restraint cone. Each end of the tubing was run outside of the exposure cage and threaded through a toggle and tightened to firmly affix the animal and prevent movement during the BOP exposure but careful to not restrict breathing. Rats were randomly assigned to sham or $36.6 \mathrm{kPa}$ blast conditions in the facing or side orientation ( $n=8$ in each condition). The sham condition had equal numbers of side and facing rats.

\section{Morris water maze task}

The water maze apparatus consisted of a blue, circular tank (74.5 $\mathrm{cm}$ deep; $180 \mathrm{~cm}$ diameter) filled with water $\left(20^{\circ} \mathrm{C}\right)$ to a depth of $60 \mathrm{~cm}$ located in a dimly lit room. A platform was submerged to a depth of $1 \mathrm{~cm}$ and placed approximately $35 \mathrm{~cm}$ from the wall of the pool in the center of the northeast quadrant. The position of the platform remained constant throughout all experiments. Blast or sham exposed rats were given four 4 block trials on a single test day. At the start of each trial, the rat is placed in the pool (snout facing the pool-wall) at one of four equally spaced starting positions: north $(\mathrm{N})$, south $(\mathrm{S})$, east $(\mathrm{E})$, and west $(\mathrm{W})$. The order of the start positions was randomized. Each rat was allowed to swim freely in the pool until it finds the hidden platform or until 90 s elapsed. If the rats did not find the platform in $90 \mathrm{~s}$ they were be manually guided to it. Once the rat found the platform it was allowed to rest for $10 \mathrm{~s}$ (reinforcement) and then removed from the pool, gently towel-dried, and placed in a holding cage (warmed by a circulating water pad) in between trials. The dependent measures for this task were (1) latency to reach the platform and (2) length of path (distance swum) to the platform averaged over the blocked trials. Spatial learning was assessed using the Noldus EthoVision XT (Noldus, Inc, Leesburg, VA, USA) video-tracking system from a camera suspended above the water maze.

\section{Immunohistochemistry}

For the immunohistochemistry portion of this study, rodents underwent 12 sessions of BOP exposure at $36.6 \mathrm{kPa}$ or sham exposure with a 24 -h interval separating each session. Animals were allowed to survive $6 \mathrm{~h}(n=3), 24 \mathrm{~h}(n=3)$, and 1 week $(n=3)$ following the final overpressure exposure session, after which they underwent transcardial perfusion with $4 \%$ paraformaldehyde. Brains were then removed and placed in fixative solution for a 24-h period. Following this post-fixation, brains were blocked and transferred to Millonig's buffer, after which they were blocked into two segments, which included: (i) brainstem/cerebellum, and (ii) bilateral hemispheres. Blocks then underwent infiltration with tissue cryoprotection solution for $48 \mathrm{~h}$, after which they were rapidly frozen in isopentane pre-cooled to $-70^{\circ} \mathrm{C}$ with dry ice. Next, $36.6 \mu \mathrm{m}$ sections were cut with a cryostat for further processing. Sectioning was performed in the sagittal plane for brainstem/cerebellum and coronal plane for bilateral hemispheres.

Semi-serial sections were washed $3 \times 10 \mathrm{~min}$ in $\mathrm{PBS}$ after which endogenous peroxidase activity was blocked with $0.5 \% \mathrm{H}_{2} \mathrm{O}_{2}$ in PBS for $30 \mathrm{~min}$. Sections were then rinsed $3 \times 10 \mathrm{~min}$ in PBS, and pre-incubated for 36.6 min with $2 \%$ Triton $\mathrm{X}$ in $10 \%$ normal goat serum (NGS) in PBS. Tissue was then incubated overnight in a 1:5,000 dilution of the APP C-terminus $1^{\circ}$ antibody (CT695, Invitrogen, Carlsbad, CA, USA). Sections were then rinsed $3 \times 10 \mathrm{~min}$ in $1 \%$ NGS in PBS and the APP C-terminus processed tissue was re-incubated in a biotinylated goat anti-rabbit $2^{\circ}$ antibody (Vector Laboratories, Burlingame, CA, USA), diluted 1:200 in 1\% NGS in PBS. The tissue was then rinsed $3 \times 10 \mathrm{~min}$ in PBS and subsequently incubated for $1 \mathrm{~h}$ with an avidin-biotin complex (ABC Standard elite kit, Vector Laboratories, Burlingame, CA, USA). After rinsing $3 \times 10 \mathrm{~min}$ in PBS and $2 \times 10 \mathrm{~min}$ in $0.1 \mathrm{M}$ phosphate buffer, the reaction product was visualized with $0.05 \%$ diaminobenzidine (DAB), $0.01 \%$ hydrogen peroxide, and $0.3 \%$ imidazole in $0.1 \mathrm{M}$ phosphate buffer for $10 \mathrm{~min}$. Sections were then mounted onto slides, dried overnight, dehydrated with alcohol/xylene, and coverslipped with mounting medium. Sections were examined with a Zeiss Axioimager Z1 microscope.

\section{Statistical analysis}

Analysis of the balance beam data was carried out with a one sided, within subjects, $t$-test. Because of the non-parametric nature of the data the assessment of group differences for the amnesia studies using the PA task utilized an overall Kruskal-Wallis analysis of variance (ANOVA) followed by Mann-Whitney $U$ tests to determine differences between specific groups. Statistical analysis of group differences in the Morris water maze (MWM) portion of the study 
was carried out using repeated measures ANOVA. Between group comparisons was accomplished with paired $t$-tests. The level of statistical significance was 0.05 . All of the behavioral experiments were performed in a blinded fashion where personnel performing the behavioral manipulations were unaware of the treatment condition.

\section{RESULTS \\ ACUTE BOP EXPOSURE}

Balance beam task

Figures 1A-C depict the effects of exposure to 36.6, 74.5, and $116.7 \mathrm{kPa} \mathrm{BOP}$, respectively, in rats performing on the balance beam task. Performance in 36.6 and $74.5 \mathrm{kPa}$ conditions showed no significant deviation of beam walking ability regardless of the time assessed after BOP exposure or orientation to the BOP wave. In contrast, rats in $116.7 \mathrm{kPa}$ side facing condition showed significantly slower latencies at the $30 \mathrm{~min}$ and $2 \mathrm{~h}$ assessments after BOP exposure while the facing condition manifested significant latency increase only at the 30-min assessment. For both the frontal and side orientation conditions the latencies to cross the beam returned to baseline levels by 2 and $6 \mathrm{~h}$, respectively, after BOP exposure and were indistinguishable from either baseline or control latencies thereafter. It should be noted that rats in the frontal and side conditions were observed to exhibit a loss of the righting reflex for durations of up to $2 \mathrm{~min}$ after the $116.7 \mathrm{kPa}$ BOP exposure.

\section{PASSIVE AVOIDANCE TASK \\ Retrograde effects}

Exposure to BOP did not produce retrograde impairment using the PA task when BOP exposure occurred within minutes after training as shown by the across the board high retention of the conditioned fear of the black (shock) compartment as shown by non-significant differences in any of the treatment conditions (Figure 2A). All rats demonstrated excellent retention by virtue of spending nearly all of their time on the white (safe) side of the black-white apparatus in a $10-\min (600 \mathrm{~s})$ test period.

\section{Anterograde effects}

Exposure to BOP produced significant anterograde impairment only in the $116.7 \mathrm{kPa}$ sideways condition and the $74.5 \mathrm{kPa}$ frontal orientation groups as shown by significant reductions in the time spent of the white side of the apparatus compared to the control

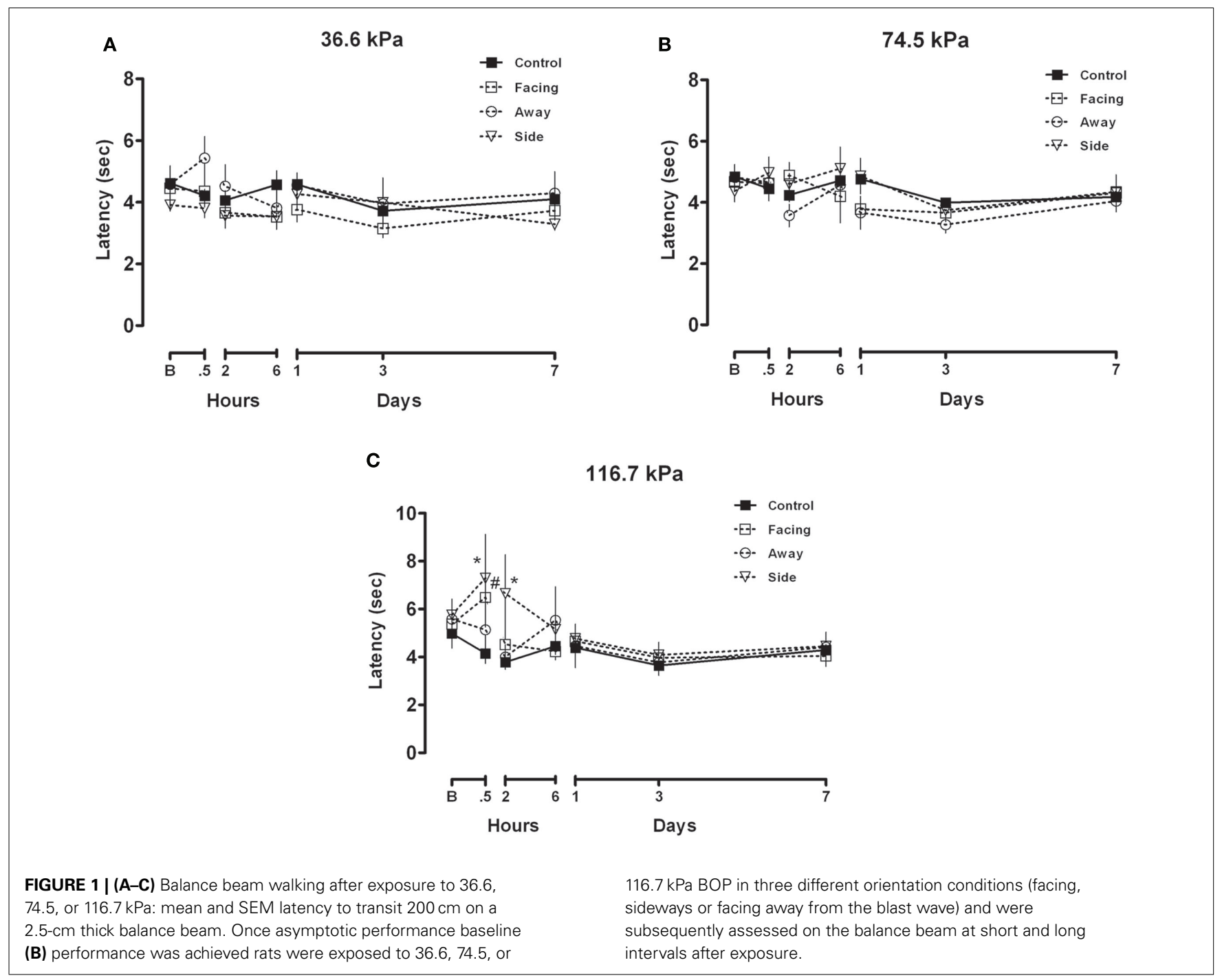




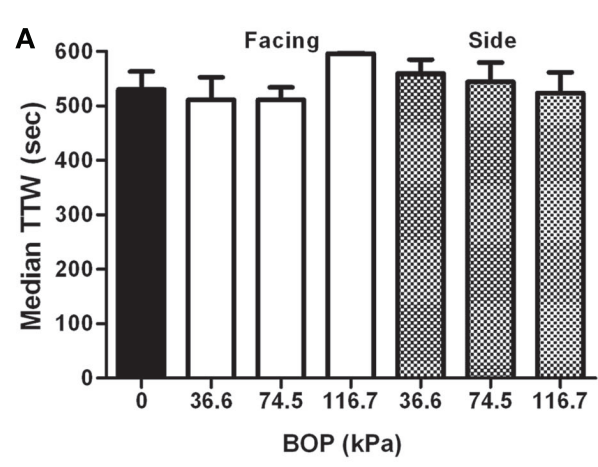

FIGURE 2 | (A) Retrograde effects of BOP: median (and standard interquartile range), total time on the white (TTW) side of the black-white shuttle box during a 10 min test session $24 \mathrm{~h}$ after passive avoidance training followed immediately by exposure to varying intensities of BOP. BOP exposed rats were either facing or sideways orientation to the blast wave. (B) Anterograde Effects of BOP: median (and standard interquartile

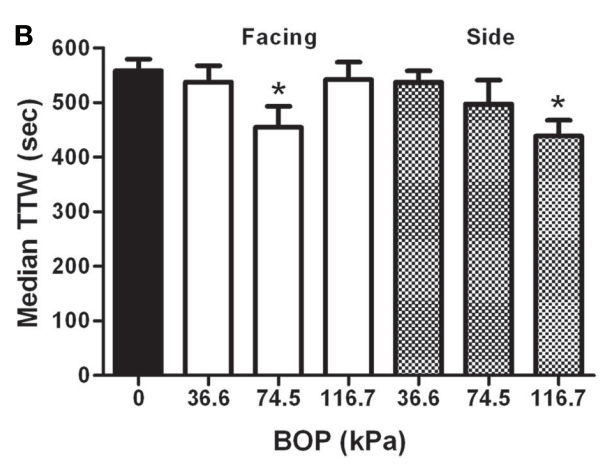

range), TTW side of the black-white shuttle box during a 10 min test session $24 \mathrm{~h}$ after passive avoidance training was administered after exposure to varying intensities of BOP. BOP exposed rats were either facing or sideways orientation to the blast wave. Significance is with a Mann-Whitney $U$ pairwise test for non-parametric data with significance criteria of $p<0.05$. condition (Figure 2B). None of the other intensity or orientation exposure conditions demonstrated evidence of memory impairment on the one-trial task.

\section{REPEATED BOP EXPOSURE}

Morris water maze task

Figure 3 depicts the latency to find the sunken platform over the course of four blocked trials in which each block represented four trials. Three conditions are depicted. The solid black line depicts the control condition that was exposed to all facets of the blast exposure procedure minus the exposure to BOP. Over the course of the blocked trials rats in the control condition reached asymptotic performance by block three. The side orientation condition to the BOP wave essentially mirrored the performance of the control group. There were no significant differences between these two treatment conditions across the blocked trials. In contrast, the group facing the BOP wave demonstrated significantly slower acquisition of the task compared to the control group. Post hoc analysis indicated that the primary differences stemmed from slower latencies to find the submerged platform in blocks two and three. By the fourth block trial the latencies for all of the groups was statistically indistinguishable.

\section{Immunohistochemistry}

Figure 4 represents composite images of APP immunohistochemistry in the $12 \mathrm{kPa} \times 36.6 \mathrm{kPa}$ condition $24 \mathrm{~h}$ after the last BOP exposure. Examination of sections processed for APP immunohistochemistry from animals exposed to 12 individual sessions of $36.6 \mathrm{kPa}$ BOP exposure and then allowed to survive $6 \mathrm{~h}, 24 \mathrm{~h}$, or 1 week following final exposure was performed. Findings were compared to SHAM injured controls prepared and allowed to survive at comparable post-preparation intervals. Within the cerebrum, brainstem, and cerebellum, there was no evidence of APP accumulation in white matter at any post-injury time point. No evidence of altered APP immunostaining was seen within cell bodies. No abnormal APP immunostaining was seen within perivascular regions. There were no appreciable differences between experimentally injured or SHAM injured controls.

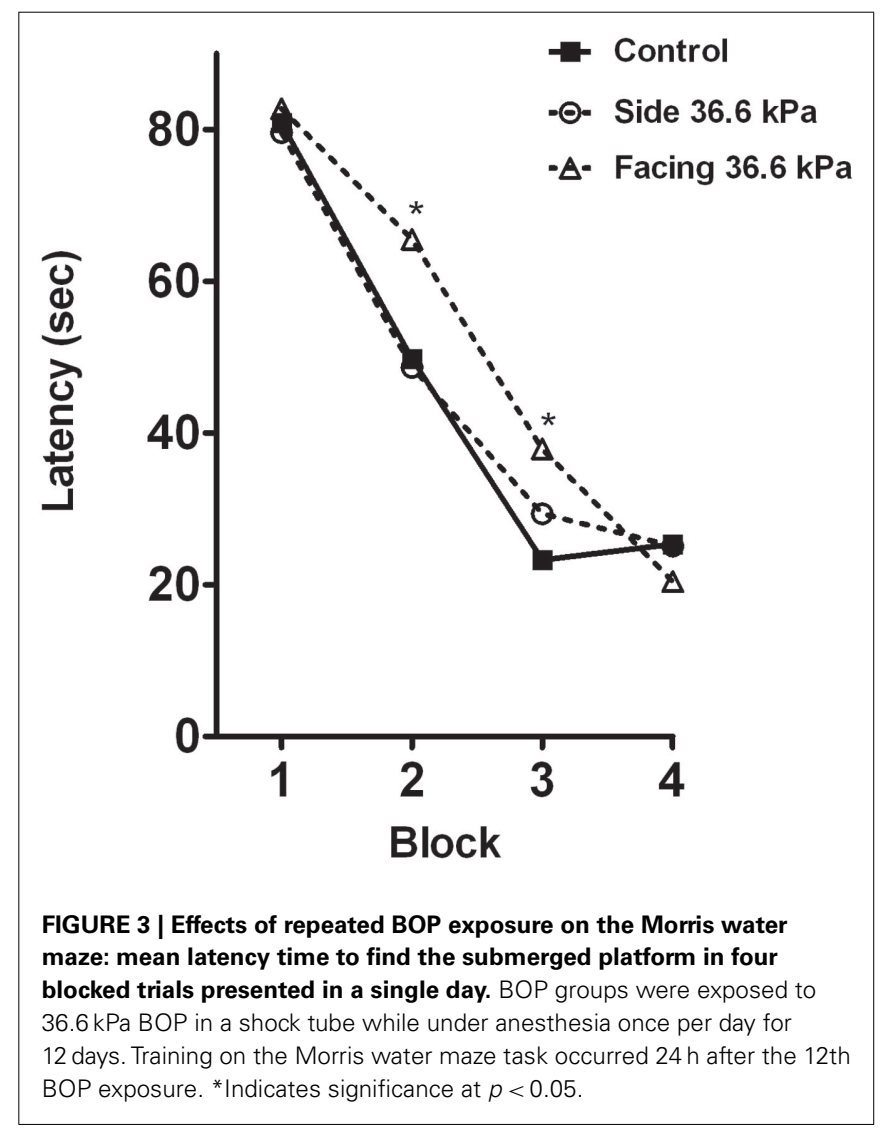

\section{DISCUSSION \\ ACUTE BOP EXPOSURE \\ Gross motor studies}

Rats exposed to $116.7 \mathrm{kPa}$ showed a transient impairment of gross motor function, which recovered after $2 \mathrm{~h}$ and was stable thereafter. This transient impairment was more severe for rats in the side orientation condition than for rats that faced the blast wave owing 


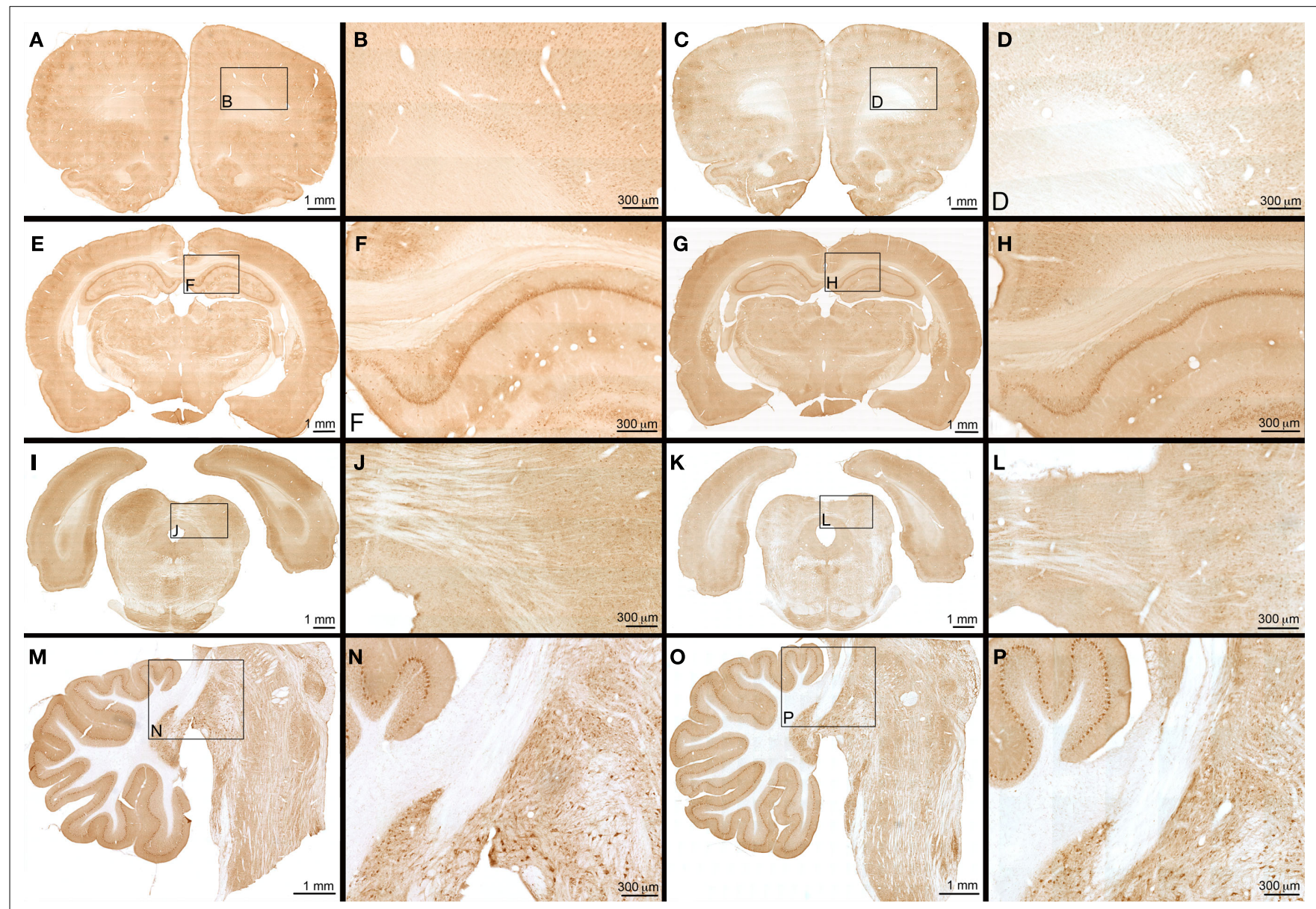

FIGURE 4 | APP immunohistochemistry - frontal overpressure exposure: APP immunohistochemistry is seen with animals exposed to 12 sessions of $36.6 \mathrm{kPa}$ blast overpressure exposure (A,B,E,F,I,J,M,N) versus SHAM injured controls $(\mathbf{C}, \mathbf{D}, \mathbf{G}, \mathbf{H}, \mathbf{K}, \mathbf{L}, \mathbf{O}, \mathbf{P})$. Photomontages of multiple photomicrographs of frontal $(\mathbf{A}, \mathbf{C})$, mid $(\mathbf{E}, \mathbf{G})$, and posterior $(\mathbf{I}, \mathbf{K})$ portions of the cerebrum are shown in coronal section. Sagittal photomicrograph of the brainstem is seen in plates $\mathbf{( M , 0 )}$. Magnifications of corresponding boxes within photomontages are seen in plates $(\mathbf{B}, \mathbf{D}, \mathbf{F}, \mathbf{H}, \mathbf{J}, \mathbf{L}, \mathbf{N}, \mathbf{P})$. Within all of the plates, no evidence of traumatic injury or difference between experimentally injured and SHAM injured controls is seen. to the observation of significant delayed latencies in the side, but not frontal, condition at the 2 -h assessment. Rats facing away from the BOP wave did not show a deficit so subsequent BOP exposure studies did not include the "facing away" condition. Importantly, the impairment observed after exposure to $116.7 \mathrm{kPa}$ BOP from a side orientation was typically associated with a transient loss of righting reflex believed to approximate an alteration or loss of consciousness (AOC or LOC). Exposure to pressure intensities less than $116.7 \mathrm{kPa}$ did not produce any impairment of gross motor function regarded as an AOC or LOC. To our knowledge this is the first demonstration of a behavioral manifestation of AOC or LOC resulting from exposure to $\mathrm{BOP}$ that is un-confounded by adverse effects of anesthesia.

\section{Amnesia studies}

Exposure to $36.6,74.5$, or $116.7 \mathrm{kPa}$ BOP within 2 min after PA training (i.e., a retrograde amnesia paradigm) did not disrupt performance when rats were tested $24 \mathrm{~h}$ after training and BOP exposure regardless of the orientation (side or frontal) to the BOP wave. This outcome is not surprising. In the retrograde paradigm the animal learns in the "normal state" which is followed by a traumatic event. It is generally assumed that the imposition of the traumatic event interferes with the consolidation process for forming a long-term memory of the learned event (McGaugh, 1966). BOP exposure would appear not to disrupt brain function to the degree necessary to produce retrograde amnesia in this paradigm.

The anterograde paradigm is typically more sensitive to disruption than the retrograde paradigm in large part because the animal is learning the event in an altered state induced by the trauma (Ahlers and Riccio, 1987). BOP exposure produced a complicated pattern whereby anterograde impairment was observed in the $116.7 \mathrm{kPa}$ sideways condition and the $74.5 \mathrm{kPa}$ frontal orientation group only. The observation of impairment in the $116.7 \mathrm{kPa}$ side group was consistent with significant impairment; rats exposed to $116.7 \mathrm{kPa}$ demonstrated a loss/alteration of consciousness and, in a small number of subjects, there was evidence of cerebral and systemic pathology (see below). We note however, that the presence of cerebral and pulmonary hemorrhage suggests some real limitations to the extrapolation of observations of outcomes with 
rodents to humans. The observed deficit in animals exposed to $74.5 \mathrm{kPa}$ facing the blast wave occurred at a BOP intensity where there was no observable impairment of motor ability as demonstrated in the balance beam task. The obvious conclusion to be drawn from these data is that AOC/LOC is not a necessary condition to exhibit amnesia from BOP exposure. Though limited by the use of only a single task to make the assessment, the apparent threshold for acute disruption of memory is $74.5 \mathrm{kPa}$. How then to explain the lack of impairment in the $116.7 \mathrm{kPa}$ frontal exposed group? This outcome would not appear to fit a clear pattern. In our studies of BOP dynamics we come to appreciate that blast is a more complex phenomenon than meets the eye. A salient feature of our work using functional outcomes together with our research on blast dynamics led by Chavko et al. (2011) clearly shows that orientation is a significant factor. We appreciate that BOP intensity, overpressure duration, and the overpressure intensity and duration function may determine differential outcome physiologically. This underscores the need to provide these key parameters in translation of research between laboratories and, of course, when contrasting physiological outcomes with native blast using real explosives. We acknowledge that peak overpressure may not be the only variable that determines the effect of exposure to BOP. For example, the observed outcome in animals impaired from a frontal or side exposure may result from the complex interplay of forces that magnify or cancel depending upon the orientation of the animal. Moreover, the forces resulting from different orientations to the blast wave may likewise result in different injury mechanisms, e.g., from increased trunk pressure when exposed sideways versus in the frontal exposure (Cernak, 2010). Similarly, protective devices such as vests may influence the manifestation of outcomes, again dependent upon the angle of engagement (orientation) to the primary blast wave and relationship to reflective waves generated around the individual and possibly inside hard "protected" surfaces.

Cerebral hemorrhage and contusions were observed in approximately $30 \%$ of the animals exposed to $116.7 \mathrm{kPa}$ (data not shown) with no apparent distinction between the side and frontal orientations. Lung hemorrhage (data not shown) was evident in all of the animals exposed to $116.7 \mathrm{kPa}$, regardless of orientation. Because the design study precluded continuation of exposure once the pathology criterion was reached, a clear limitation of this study is that we have insufficient data to fully characterize the pathologic outcome. There are a significant number of studies that have exposed (anesthetized) rats to BOPs in the range of approximately 17 psi $(116.7 \mathrm{kPa})$ and higher. Many of these report only the effects on the brain. Comparison is also complicated by other factors such as whether the animal was completely in the blast tube or outside of it, or whether the overpressure wave was administered directly to the head region. Our data (Chavko et al., 2007), and data of our colleagues using whole animal exposure to BOP where the animal was completely in the chamber have shown pathology, primarily hemorrhage and inflammation, in lung, gut, heart, liver, kidney, and brain (Dalle Luca, unpublished observations). As a consequence, exposure to $116.7 \mathrm{kPa}$ in our laboratory which produces evidence of traumatic brain injury as reflected by behavioral and pathologic changes, and occurs in a context of a polytrauma event to the whole animal. At this level of BOP intensity, multiple indices of trauma in many organ systems complicates interpretation of a specific effect of BOP on brain functioning specifically (Cernak, 2010). Yet another complicating factor to the interpretation of the data is the relative contribution of primary blast wave effects and those associated with movement produced by the blast wave, tertiary blast effects. The latter would involve acceleration and deceleration forces and potential for blunt force trauma that could directly damage tissue, particularly at higher intensities of BOP. We acknowledge that the behavioral and pathological outcome measures we observed are the result of both primary and tertiary blast wave forces. On the one hand the combination of primary and tertiary forces is valid, representing the dynamics involved when military personnel are exposed to IEDs on the battlefield. On the other hand it underscores the need to precisely determine the contributions of the various forces to the functional and pathological outcomes resulting from exposure to BOP and IEDs.

\section{Synthesis from acute BOP exposure}

When taken together the behavioral and pathological data from this series of studies provide the conditions where acute exposure to BOP precipitates the manifestations of TBI across the severity spectrum from mild to moderate/severe TBI. Exposure to $116.7 \mathrm{kPa}$ produces $\mathrm{AOC} / \mathrm{LOC}$ and anterograde amnesia, thus meeting three of the four criteria for the clinical definition of mTBI. A caveat to the conclusion that exposure to $116.7 \mathrm{kPa}$ produces mTBI stems from the observation that $116.7 \mathrm{kPa}$ is close to a threshold for cerebral hemorrhage as well. In $30 \%$ of subjects exposure to $116.7 \mathrm{kPa}$ produces moderate to severe TBI as evidenced by cerebral damage. In the remaining $70 \%$ of subjects exposed to $116.7 \mathrm{kPa}$ there is no evidence of cerebral pathology with the accompanying behavioral (cognitive and motor) impairment. This latter observation would appear to fulfill the criteria for mTBI in which there is demonstrable cognitive impairment in the absence of any observable pathology. A complicating factor in this analysis is the observation of lung pathology in all animals exposed to $116.7 \mathrm{kPa}$ and the observation by others that many other organs show damage. Lung pathology alone was reason enough to make the use of repeated exposure to $116.7 \mathrm{kPa} \mathrm{BOP}$ for the repeated exposure experiments untenable. Our observation of functional impairment coupled with clear evidence of a neuropathological outcome, albeit non-fatal, after a single acute exposure at the $116.7 \mathrm{kPa}$ intensity is consistent with other investigators who have noted similar patterns at pressures ranging from $116.7 \mathrm{kPa}$ and significantly higher (Long et al., 2009; Cernak et al., 2010; Risling et al., 2010; Svetlov et al., 2010; Garman et al., 2011; Lu et al., 2011).

We note some potential limitations to this study. The use of the balance beam task is a crude measure of motor ability and does not necessarily reflect the most sensitive measure to conclude that cognitive activity was unaffected after BOP exposure or to conclude an absence of an alteration in consciousness. There could be, and likely is, impairment of cognitive performance at the lower BOP intensities; an observation born out by the anterograde impairment in the $74.5 \mathrm{kPa}$ facing condition. In addition, the shock tube that we use limits the interpretation of our data. Clearly, further study to assess cognition in the immediate 
post-blast period using more sensitive measures is warranted, particularly if impairment around the traumatic event is important for the manifestation of long-term deficits as well as studies that vary overpressure duration exposure and parametrically change the shape, and presumably the physiological consequences resulting from the overpressure exposure. Our studies with the breacher populations exposed to multiple blast events would not support a contention that an alteration in consciousness is a necessary condition for the manifestation of a long-term impairment. In our study of (protected) breachers we observed a peak pressure recorded from helmet-mounted pressure probes of $88.9 \mathrm{kPa}$ (12.9 psi). Our observation of a slight impairment memory at $74.5 \mathrm{kPa}$ is within the upper region of exposure intensities seen in military populations.

The small, but significant, deficit in the $74.5 \mathrm{kPa}$ facing toward the blast wave is intriguing on two fronts: one, the deficit was noted at a BOP intensity that did not manifest any observable pathology, and two, it was not associated with an alteration in consciousness as measured by the balance beam performance. This is perhaps, the clearest and most unequivocal demonstration of a mTBI symptom (anterograde amnesia) that meets the clinical criteria and is not confounded by the presence of cerebral or systemic pathology. This subtle deficit occurred in the absence of any observable pathology in the brain or any evidence of systemic pathology, e.g., injury to the lung or other organs that was observed acutely with exposure to $116.7 \mathrm{kPa} \mathrm{BOP}$.

\section{REPEATED BOP EXPOSURE}

\section{Morris water maze}

Repeated exposure to $36.6 \mathrm{kPa}$ BOP resulted in a slight, but significant, retardation of acquisition of the spatial learning task but only in the rats facing the BOP wave. The impairment was observed in the second and third blocks trials where rats exposed to BOP in the facing condition was significantly slower to locate the submerged platform relative to the $36.6-\mathrm{kPa}$ side exposure and sham exposure conditions. The acquisition impairment was transient since by the fourth block of trials all animals reached an asymptotic level of performance. The observation of impairment on a water maze task after exposure to low levels of BOP is consistent with the observations of Saljo et al. who demonstrated impairment with three exposures $20 \mathrm{~min}$ apart at BOP intensities of 10 and $30 \mathrm{kPa}$. Further, Saljo noted that the impairments were associated with increases in intracranial pressure (Saljo et al., 2009).

\section{APP immunohistochemistry}

Examination of sections processed for APP immunohistochemistry from animals exposed to 12 individual sessions of $36.6 \mathrm{kPa}$ BOP exposure and then allowed to survive $6 \mathrm{~h}, 24 \mathrm{~h}$, or 1 week following final exposure was performed. Findings were compared to SHAM injured controls prepared and allowed to survive at comparable post-preparation intervals. Within the cerebrum, brainstem, and cerebellum, there was no evidence of APP accumulation in white matter at any post-injury time point. No evidence of altered APP immunostaining was seen within cell bodies. No abnormal APP immunostaining was seen within perivascular regions. There were no appreciable differences between experimentally injured or SHAM injured controls.

\section{Synthesis from repeated BOP exposure}

Our observations of an impairment of performance in the water maze after repeated exposure to BOP adds to a growing body of work to understand the effects of repeated exposure to blast events in a manner similar to that reported in deployed military personnel on the battlefield as well as observations of military breacher populations experiencing cognitive impairments. Initially we hypothesized that functional deficits after repeated blast events would result from impaired functioning of white matter in the brain. This hypothesis was not supported based on the observation that APP accumulation was unchanged in rats at $6 \mathrm{~h}, 1$ day, and 7 days post BOP exposure, no changes in APP accumulation were observed. In our experimental preparation we went to great lengths to disallow movement resulting from the BOP exposure that would result in acceleration or deceleration rotational forces that could produce white matter injury as shown by positive APP staining, however, since we did not employ accelerometers in our animals during the BOP exposure we cannot rule out a contribution of movement of the animal in contributing to the behavioral manifestations we observed. Risling et al. (2010) observed functional deficits in rats exposed to a primary blast wave at levels much higher than used here $(136-260 \mathrm{kPa})$. We acknowledge that the absence of changes in APP immunohistochemistry reflecting impaired flow through the axon secondary to structural breakdown of the axonal transport system does not by itself permit a conclusion that axons are unaffected by exposure to low levels of a primary BOP wave. Subtle functional changes in memory like those observed in this study could result from impaired receptor, ion channel, or other metabolic functioning, e.g., depletion of mitochondria, in white or gray matter that could account for changes in cognitive processing. These changes may not necessary culminate in breakdown of the axonal transport mechanism, leading to APP accumulation. Interestingly, an emerging picture is beginning to develop where exposure to a primary blast wave and low levels of BOP produces downregulation of genes and indices of inflammatory changes (Risling et al., 2010; Saljo et al., 2010b; Pun et al., 2011). An understanding of the physiological underpinnings of $\mathrm{mTBI}$ resulting from blast may yet require more sensitive techniques to elucidate the physiological changes that are responsible for the functional changes (Agoston et al., 2009). Importantly, the physiological and biochemical changes occurring after repeated exposure to BOP in the lung, e.g., lowered pulmonary tolerance (Dodd et al., 1990), could contribute to systemic and CNS pathophysiology, though in our studies we have observed no evidence of overt lung pathology resulting from repeated BOP exposure.

\section{CONCLUSION}

There are several key findings in the current study. Consideration of the outcome of the acute and repeated BOP exposures clearly indicate BOP intensity, and orientation to the BOP wave, are important variables influencing functional and pathologic outcome (Cernak et al., 2010). Although much more work clearly is needed to understand the direct effects of BOP exposure on brain tissue, our observation that orientation to the BOP wave can significantly influence the biological effects of BOP are supported by the extensive series of studies of Chavko et al. (2007, 2011). In these studies the pressure wave recorded in front position had 
Table 2 | Proposed dichotomy of long-term blast injuries: the table depicts characteristics of two distinct blast injury patterns based upon experimental, clinical, and operational observations.

\begin{tabular}{lll}
\hline Dimension & Type 1 & Type 2 \\
\hline Blast frequency & Single & Single or multiple \\
Blast intensity & $120 \mathrm{kPa}+$ & $\leq 75 \mathrm{kPa}$ \\
Physical forces & 1 blast wave, 2 penetrating, 3 acceleration/deceleration & 1 Blast wave \\
Clinical manifestations & Mild-severe TBI, PCS/PTSD "polytrauma" & Mild TBI/PCS/PTSD "subclinical" \\
Clinical onset & Event-related symptoms & Insidious onset over time \\
Radiology/pathology & CT/MRI hemorrhage, inflammation, vasospasm, edema, & No conventional signal, DTI, fMRI, MRS (TBD), white \\
& white/gray matter damage & matter injury? \\
Biomarkers & Inflammatory & GFAP, UCH-L1
\end{tabular}

higher amplitude, faster rise time, and longer duration than the pressure wave from the side-on and head-off exposures. The results of these studies indicated orientation-specific dynamics whereby higher shock wave amplitude in the brain as well as differences in the duration of the shock wave was observed with respect to the orientation of the head to blast, particularly with the head-on exposure. The head-on exposure was preferentially affected when exposed to $74.5 \mathrm{kPa}$ in the acute experiment and repeated exposure to twelve $36.6 \mathrm{kPa}$ was also preferentially affected compared to the side orientation. The differential functional changes manifested by different orientations to the BOP likely result from specific biochemical, physiological or gene changes in specific brain regions, for example the brainstem areas identified by recent investigations in military personnel (Mac Donald et al., 2011) or in animal models (Garman et al., 2011).

\section{PROPOSED DICHOTOMY OF LONG-TERM BLAST INJURY}

In the course of our pursuit to develop an animal model of the breacher type impairment, that is, an impairment of cognition that occurs with repeated exposure to low-level blast events over a protracted timeframe, we have arrived at a conclusion that there are potentially at least two types of blast TBI manifested in military personnel where symptoms endure well after transient disruptions, i.e., acute mTBI, are noted. These types are presented in Table 2. Considering the clinical manifestations and operational observations together with a broad swath of studies to assess blast in animal models, it appears that blast injuries to the brain appear to manifest as two distinct types. Type 1 blast brain injury results from a single, intense ( $116.7 \mathrm{kPa}$ or higher), event involving primary and tertiary physical forces primarily but may also include secondary forces as well. These latter forces may induce penetrating trauma. Clinically this type of brain injury is one component of injury to a number of organ systems that could include limb amputations in the extreme. Moderate to severe TBI consequent with detectable physical disruption of brain tissue would be detectible using standard diagnostic imaging techniques. Many of these casualties could manifest cerebral vasospasm so well characterized by Armonda et al. (2006) in TBI patients seen at Bethesda Naval Hospital. Biomarkers of brain and systemic injury would likely be elevated levels of cytokines and chemokines in serum and cerebral spinal fluid reflecting multi-system polytrauma. These manifestations would be the result of a single blast event. In contrast, Type 2 brain injury that results from multiple, low intensity blast exposures. Presumably this manifestation represents a primary blast event devoid of acceleration/deceleration forces that would accompany an intense blast event (Risling et al., 2010). The primary behavioral outcome upon presentation to a diagnostician is a post-concussive syndrome that may, or may not, have resulted from an explicit mTBI experienced around a single blast event. This deficit appears to be protracted, lasting for weeks or months after the service member has returned to duty stateside. Clinical radiological assessment is typically negative using standard CT/MRI methods and newer analysis modalities, for example, DTI (Levin et al., 2010; Mac Donald et al., 2011), would appear to hold some promise of localizing deficits in axonal bundles stretched by the blast event.

Our goal at the outset of our research was to develop an animal model of type 2 blast injury, in part based upon our work with military breacher populations who reported cognitive impairment after extended experience with blast events operationally. In our pursuit of this animal model of repeated blast exposure we systematically assessed the effects of blast exposure in a dose-response, dose-escalating fashion. We determined, systematically, the BOP intensities that result in impairments linked to specific acute mTBI and severe TBI symptoms. Blast-induced TBI that was moderate to severe was associated with overt indices of pathology (hemorrhage and inflammation) in the brain, lung, and other organs. We believe that a single exposure to a blast event that produces polytrauma to multiple organ systems is a distinct entity, a type one TBI, relative to the TBI that results from repeated exposures. Repeated exposure to low intensity BOP that did not induce white matter damage or overt pathology produced an impairment of spatial memory that was again, specific to the orientation to the BOP wave. The latter observation would appear to fulfill the criteria for a type 2 TBI. Further investigation will no doubt shed light on the underlying mechanisms and the types of TBI that result from exposure(s) to blast.

\section{ACKNOWLEDGMENTS}

The authors are extremely grateful to colleagues Frank Tortella, Joe Long, and Richard Bauman for their assistance in making this project a reality. We are indebted to $\mathrm{Dr}$. Fu $\mathrm{Du}$ and his colleagues for their consistently high quality immunohistochemistry. This work was supported by the Office of Naval Research: 0601153N.0000.000A0702 to STA. Animal experiments (approved protocol K03-07) were conducted according to the principles set 
forth in the Guide for the Care and Use of Laboratory Animals, Institute of Laboratory Animals Resources, National Research Council, National Academy Press, 1996. The opinions contained herein are those of the authors and are not to be construed as official or reflecting the views of the Department of the Navy or the

\section{REFERENCES}

Agoston, D. V., Gyorgy, A., Eidelman, O., and Pollard, H. B. (2009). Proteomic biomarkers for blast neurotrauma: targeting cerebral edema, inflammation, and neuronal death cascades. J. Neurotrauma 26, 901-911.

Ahlers, S. T., and Riccio, D. C. (1987). Anterograde amnesia induced by hyperthermia in rats. Behav. Neurosci. 101, 333-340.

Armonda, R. A., Bell, R. S., Vo, A. H., Ling, G., Degraba, T. J., Crandall, B., Ecklund, J., and Campbell, W. W. (2006). Wartime traumatic cerebral vasospasm: recent review of combat casualties. Neurosurgery 59, 1215-1225; discussion 1225.

Bell, R. S., Vo, A. H., Neal, C. J., Tigno, J., Roberts, R., Mossop, C., Dunne, J. R., and Armonda, R. A. (2009). Military traumatic brain and spinal column injury: a 5-year study of the impact blast and other military grade weaponry on the central nervous system. J. Trauma. 66, S104-S111.

Cernak, I. (2010). The importance of systemic response in the pathobiology of blast-induced neurotrauma. Front. Neurol. 1:151. doi:10.3389/fneur.2010.00151

Cernak, I., Merkle, A. C., Koliatsos, V. E., Bilik, J. M., Luong, Q. T., Mahota, T. M., Xu, L., Slack, N., Windle, D., and Ahmed, F. A. (2010). The pathobiology of blast injuries and blastinduced neurotrauma as identified using a new experimental model of injury in mice. Neurobiol. Dis. 41, 538-551.

Cernak, I., Wang, Z., Jiang, J., Bian, X., and Savic, J. (2001). Cognitive deficits following blast injuryinduced neurotrauma: possible involvement of nitric oxide. Brain Inj. 15, 593-612.

Chavko, M., Koller, W. A., Prusaczyk, W. K., and Mccarron, R. M. (2007). Measurement of blast wave by a miniature fiber optic pressure transducer in the rat brain. J. Neurosci. Methods 159, 277-281.

Chavko, M., Watanabe, T., Adeeb, S., Lankasky, J., Ahlers, S. T., and Mccarron, R. M. (2011). Relationship between orientation to a blast and pressure wave propagation inside the rat brain. J. Neurosci. Methods 195, 61-66.

Dodd, K. T., Yelverton, J. T., Richmond, D. R., Morris, J. R., and Ripple, G. R. (1990). Nonauditory injury threshold for repeated intense freefield impulse noise. J. Occup. Med. 32, 260-266.

Elder, G. A., Mitsis, E. M., Ahlers, S. T., and Cristian, A. (2010). Blast-induced mild traumatic brain injury. Psychiatr. Clin. North Am. 33, 757-781.

French, L. M., and Parkinson, G. W. (2008). Assessing and treating veterans with traumatic brain injury. $J$. Clin. Psychol. 64, 1004-1013.

Galarneau, M. R., Woodruff, S. I., Dye, J. L., Mohrle, C. R., and Wade, A. L. (2008). Traumatic brain injury during Operation Iraqi Freedom: findings from the United States Navy-Marine Corps Combat Trauma Registry. J. Neurosurg. 108, 950-957.

Garman, R. H., Jenkins, L. W., Switzer, R. C. III, Bauman, R. A., Tong, L. C., Swauger, P. V., Parks, S. A., Ritzel, D. V., Dixon, C. E., Clark, R. S., Bayir, H., Kagan, V., Jackson, E. K., and Kochanek, P. M. (2011). Blast exposure in rats with body shielding is characterized primarily by diffuse axonal injury. J. Neurotrauma $28,947-959$.

Gaylord, K. M., Cooper, D. B., Mercado, J. M., Kennedy, J. E., Yoder, L. H., and Holcomb, J. B. (2008). Incidence of posttraumatic stress disorder and mild traumatic brain injury in burned service members: preliminary report. J. Trauma. 64, S200S205; discussion S205-S206.

Hicks, R. R., Fertig, S. J., Desrocher, R. E., Koroshetz, W. J., and Pancrazio, J. J. (2010). Neurological effects of blast injury. J. Trauma. 68, 1257-1263.

Hoge, C. W., Mcgurk, D., Thomas, J. L., Cox, A. L., Engel, C. C., and Castro, C. A. (2008). Mild traumatic brain injury in U.S. Soldiers returning from Iraq. N. Engl. J. Med. 358, 453-463.

Kennedy, J. E., Cullen, M. A., Amador, R. R., Huey, J. C., and Leal, F. O. (2010a). Symptoms in military service members after blast mTBI with and without associated injuries. Neurorehabilitation 26, 191-197.

naval service at large. Animal experiments were conducted according to the principles set forth in the Guide for the Care and Use of Laboratory Animals at the WRAIR/NMRC AAALAC-approved facility and was approved by the WRAIR/NMRC Institutional Animal Care and Use Committee (IACUC).

Kennedy, J. E., Leal, F. O., Lewis, J. D., Cullen, M. A., and Amador, R. R. (2010b). Posttraumatic stress symptoms in OIF/OEF service members with blast-related and non-blastrelated mild TBI. NeuroRehabilitation 26, 223-231.

Levin, H. S., Wilde, E., Troyanskaya, M., Petersen, N. J., Scheibel, R., Newsome, M., Radaideh, M., Wu, T., Yallampalli, R., Chu, Z., and Li, X. (2010). Diffusion tensor imaging of mild to moderate blastrelated traumatic brain injury and its sequelae. J. Neurotrauma 27, 683-694.

Lew, H. L., Otis, J. D., Tun, C., Kerns, R. D., Clark, M. E., and Cifu, D. X. (2009). Prevalence of chronic pain, posttraumatic stress disorder, and persistent postconcussive symptoms in OIF/OEF veterans: polytrauma clinical triad. J. Rehabil. Res. Dev. 46, 697-702.

Lippa, S. M., Pastorek, N. J., Benge, J. F., and Thornton, G. M. (2010). Postconcussive symptoms after blast and nonblast-related mild traumatic brain injuries in Afghanistan and Iraq war veterans. J. Int. Neuropsychol. Soc. 16, 856-866.

Long, J. B., Bentley, T. L., Wessner, K. A., Cerone, C., Sweeney, S., and Bauman, R. A. (2009). Blast overpressure in rats: recreating a battlefield injury in the laboratory. J. Neurotrauma 26 , 827-840.

Lu, J., Ng, K. C., Ling, G. S., Wu, J., Poon, J. F., Kan, E. M., Tan, M. H., Wu, Y. J., Li, P., Moochhala, S., Yap, E., Lee, L. K., Teo, A. L., Yeh, I. B., Sergio, D. M., Chua, F., Kumar, S. D., and Ling, E. A. (2011). Effect of blast exposure on the brain structure and cognition in the Macaca fascicularis. J. Neurotrauma. PMID: 21639720. [Epub ahead of print].

Mac Donald, C. L., Johnson, A. M. Cooper, D., Nelson, E. C., Werner, N. J., Shimony, J. S., Snyder, A. Z., Raichle, M. E., Witherow, J. R., Fang, R., Flaherty, S. F., and Brody, D. L. (2011). Detection of blast-related traumatic brain injury in U.S. military personnel. N. Engl. J. Med. 364, 2091-2100.

McGaugh, J. L. (1966). Time-dependent processes in memory storage. Science 153, 1351-1358.
Moochhala, S. M., Md, S., Lu, J., Teng, C. H., and Greengrass, C. (2004). Neuroprotective role of aminoguanidine in behavioral changes after blast injury. J. Trauma. 56, 393-403.

Moore, D. F., and Jaffee, M. S. (2010). Military traumatic brain injury and blast. NeuroRehabilitation 26, 179-181.

Mora, A. G., Ritenour, A. E., Wade, C. E., Holcomb, J. B., Blackbourne, L. H., and Gaylord, K. M. (2009). Posttraumatic stress disorder in combat casualties with burns sustaining primary blast and concussive injuries. J. Trauma. 66, S178-S185.

Okie, S. (2005). Traumatic brain injury in the war zone. N. Engl. J. Med. 352, 2043-2047.

Peskind, E. R., Petrie, E. C., Cross, D. J., Pagulayan, K., Mccraw, K., Hoff, D., Hart, K., Yu, C. E., Raskind, M. A., Cook, D. G., and Minoshima, S. (2010). Cerebrocerebellar hypometabolism associated with repetitive blast exposure mild traumatic brain injury in 12 Iraq war Veterans with persistent post-concussive symptoms. Neuroimage 54(Suppl. 1), S76-S82.

Pun, P. B., Kan, E. M., Salim, A., Li, Z., Ng, K. C., Moochhala, S. M., Ling, E. A., Tan, M. H., and Lu, J. (2011). Low level primary blast injury in rodent brain. Front. Neurol. 2:19. doi:10.3389/fneur.2011.00019

Risling, M., Plantman, S., Angeria, M., Rostami, E., Bellander, B. M., Kirkegaard, M., Arborelius, U., and Davidsson, J. (2010). Mechanisms of blast induced brain injuries, experimental studies in rats. Neuroimage 54(Suppl. 1), S89-S97.

Saljo, A., Bolouri, H., Mayorga, M., Svensson, B., and Hamberger, A. (2010a). Low-level blast raises intracranial pressure and impairs cognitive function in rats: prophylaxis with processed cereal feed. J. Neurotrauma 27, 383-389.

Saljo, A., Mayorga, M., Bolouri, H., Svensson, B., and Hamberger, A (2010b). Mechanisms and pathophysiology of the low-level blast brain injury in animal models. $\mathrm{Neu}$ roimage 54(Suppl. 1), S83-S88. 
Saljo, A., Svensson, B., Mayorga, M., Hamberger, A., and Bolouri, H. (2009). Low-level blasts raise intracranial pressure and impair cognitive function in rats. J. Neurotrauma 26, 1345-1352.

Snell, F. I., and Halter, M. J. (2010). A signature wound of war: mild traumatic brain injury. J. Psychosoc. Nurs. Ment. Health Serv. 48, 22-28.

Svetlov, S. I., Prima, V., Kirk, D. R., Gutierrez, H., Curley, K. C., Hayes, R. L., and Wang, K. K. (2010). Morphologic and biochemical characterization of brain injury in a model of controlled blast overpressure exposure. J. Trauma. 69, 795-804.
Warden, D. (2006). Military TBI during the Iraq and Afghanistan wars. J. Head Trauma Rehabil. 21, 398-402.

Warden, D. L., and French, L. (2005). Traumatic brain injury in the war zone. N. Engl. J. Med. 353, 633-634.

Wilk, J. E., Thomas, J. L., Mcgurk, D. M., Riviere, L. A., Castro, C. A., and Hoge, C. W. (2010). Mild traumatic brain injury (concussion) during combat: lack of association of blast mechanism with persistent postconcussive symptoms. J. Head Trauma Rehabil. 25, 9-14.

Zouris, J. M., Walker, G. J., Dye, J., and Galarneau, M. (2006). Wounding patterns for U.S. marines and sailors during Operation Iraqi Freedom, major combat phase. Mil. Med. 171 246-252.

Conflict of Interest Statement: The authors declare that the research was conducted in the absence of any commercial or financial relationships that could be construed as a potential conflict of interest.

Received: 13 October 2011; accepted: 16 February 2012; published online: 05 March 2012.

Citation: Ahlers ST, Vasserman-Stokes E, Shaughness MC, Hall AA, Shear DA, Chavko M, McCarron RM and Stone $J R$ (2012) Assessment of the effects of acute and repeated exposure to blast overpressure in rodents: toward a greater understanding of blast and the potential ramifications for injury in humans exposed to blast. Front. Neur. 3:32. doi 10.3389/fneur.2012.00032

This article was submitted to Frontiers in Neurotrauma, a specialty of Frontiers in Neurology.

Copyright (c) 2012 Ahlers, VassermanStokes, Shaughness, Hall, Shear, Chavko, McCarron and Stone. This is an openaccess article distributed under the terms of the Creative Commons Attribution Non Commercial License, which permits non-commercial use, distribution, and reproduction in other forums, provided the original authors and source are credited. 